\title{
Casimir effect in deformed field
}

\author{
I. O. Vakarchuk \\ Department for Theoretical Physics, \\ Ivan Franko National University of Lviv \\ 12 Drahomanov St., Lviv, UA-79005, Ukraine
}

December 16, 2018

\begin{abstract}
The Casimir energy is calculated in one-, two-, and three-dimensional spaces for the field with generalized coordinates and momenta satisfying the deformed Poisson brackets leading to the minimal length.

Key words: Casimir energy, deformation parameter, minimal length.

PACS numbers: 03.70.+k, 12.20.-m,

\section{Initial equations}

The Casimir effect, starting from the seminal work [1], has been studied in the course of several decades for various physical systems from the problems of solid state physics to those of cosmology. Recently, some interest arose in the study of this effect in the so-called deformed spaces, i. e., in the spaces with deformed Poisson brackets and, in particular, in those leading to the minimal lengths [2, 3, 4.

In our work, we will study the Casimir effect for the deformed electromagnetic field with the Hamiltonian

$$
\hat{H}=\sum_{\mathbf{k}} \sum_{\alpha}\left(\frac{\hat{P}_{\mathbf{k}, \alpha}^{2}}{2}+\frac{\omega_{k}^{2} \hat{Q}_{\mathbf{k}, \alpha}^{2}}{2}\right),
$$

where $\mathbf{k}$ is the wave vector, $\alpha$ is the polarization index, the frequency $\omega_{k}=c k$, $c$ is the speed of light in a vacuum, $\hat{Q}_{\mathbf{k}, \alpha}, \hat{P}_{\mathbf{k}, \alpha}$ are the operators of generalized coordinates and momenta that satisfy the deformed Poisson brackets:

$$
\hat{Q}_{\mathbf{k}, \alpha} \hat{P}_{\mathbf{k}, \alpha}-\hat{P}_{\mathbf{k}, \alpha} \hat{Q}_{\mathbf{k}, \alpha}=i \hbar\left(1+\beta \hat{P}_{\mathbf{k}, \alpha}^{2}\right),
$$

$\beta \geq 0$ is the deformation parameter, all the other commutators equal zero. Let us also note that the deformation parameter can depend on $\mathbf{k}$ and $\alpha$. In this work, $\beta$ is put constant.
\end{abstract}


As is known, such commutation relations lead to the existence of the minimal length $\sqrt{\left\langle\hat{Q}_{\mathbf{k}, \alpha}\right\rangle^{2}}=\hbar \sqrt{\beta}$ in the space of field coordinates [5]. Obviously, in this case one has not the deformation of a real space but that of the field itself. Proceeding to new operators $\hat{q}_{\mathbf{k}, \alpha}, \hat{p}_{\mathbf{k}, \alpha}$,

$$
\hat{q}_{\mathbf{k}, \alpha}=\hat{Q}_{\mathbf{k}, \alpha}, \quad \hat{P}_{\mathbf{k}, \alpha}=\frac{1}{\sqrt{\beta}} \tan \left(\hat{p}_{\mathbf{k}, \alpha} \sqrt{\beta}\right),
$$

it is easy to show that they are canonically conjugated,

$$
\hat{q}_{\mathbf{k}, \alpha} \hat{p}_{\mathbf{k}, \alpha}-\hat{p}_{\mathbf{k}, \alpha} \hat{q}_{\mathbf{k}, \alpha}=i \hbar
$$

and the Hamiltonian equals

$$
\hat{H}=\sum_{\mathbf{k}} \sum_{\alpha}\left(\frac{\omega_{k}^{2} \hat{q}_{\mathbf{k}, \alpha}^{2}}{2}+\frac{\tan ^{2}\left(\hat{p}_{\mathbf{k}, \alpha} \sqrt{\beta}\right)}{2 \beta}\right) .
$$

The equations of motion of the field were studied in [6], where the Hamiltonian was presented in the form of the expansion over the powers of ordinary creation and annihilation operators. Thus, the field equations are non-linear and, generally speaking, might be analyzed, as a rule, by means of the perturbation theory. A non-linear field described by $q$-oscillators was studied in [7.

The energy levels of the harmonic oscillator Hamiltonian (5) with the commutation relations (2) are well known [5, 8]. Therefore, the energy levels of the deformed field are as follows:

$$
\begin{aligned}
E_{\ldots, N_{\mathbf{k}, \alpha}, \ldots} & =\sum_{\mathbf{k}} \sum_{\alpha} \hbar \omega_{k}\left[\left(N_{\mathbf{k}, \alpha}+\frac{1}{2}\right) \sqrt{1+\left(\frac{\beta \hbar \omega_{k}}{2}\right)^{2}}\right. \\
& \left.+\frac{\beta \hbar \omega_{k}}{2}\left(N_{\mathbf{k}, \alpha}^{2}+N_{\mathbf{k}, \alpha}+\frac{1}{2}\right)\right]
\end{aligned}
$$

where the quantum numbers $N_{\mathbf{k}, \alpha}=0,1,2, \ldots$.

The energy of the vacuum state of the field, when $N_{\mathbf{k}, \alpha}=0$, equals

$$
E_{\ldots, 0, \ldots}=\sum_{\mathbf{k}} \sum_{\alpha} \frac{\hbar \omega_{k}}{2}\left(\sqrt{1+\left(\frac{\beta \hbar \omega_{k}}{2}\right)^{2}}+\frac{\beta \hbar \omega_{k}}{2}\right) .
$$

The aim of the present work is the calculation of the Casimir energy for the deformed space as a function of the deformation parameter $\beta$ proceeding from Eq. (7).

\section{Casimir energy in a one-dimensional space}

Let the scalar field be concentrated in a one-dimensional (1D) space on the segment of length $a$ along the $x$ axis between the points $x=0$ and $x=a$, in 
which it equals zero. At such conditions, the wave vector in Eq. (7) $k=\pi n / a$, $n=1,2, \ldots$ and there is no polarization. By definition, the Casimir energy $\varepsilon$ equals the difference of the energy density (7) of a vacuum in volume $a$ and that in an infinite volume:

$$
\begin{aligned}
\varepsilon & =\frac{1}{a^{2}} \sum_{n=1}^{\infty} \frac{\hbar c \pi n}{2}\left(\sqrt{1+\left(\frac{\beta \hbar c}{2 a} n\right)^{2}}+\frac{\beta \hbar c}{2 a} n\right) \\
& -\frac{1}{2 \pi} \int_{-\infty}^{\infty} \frac{\hbar c|k|}{2}\left(\sqrt{1+\left(\frac{\beta \hbar c}{2} k\right)^{2}}+\frac{\beta \hbar c}{2}|k|\right) d k .
\end{aligned}
$$

To regularize this expression one can introduce a cut-off function $e^{-\nu k}, \nu>0$, which ensures both the convergence of the summation over $n$ and the integration over $k$. Further we make the change of the variable of integration $k=\pi n / a$ and get as a result:

$$
\begin{aligned}
\varepsilon \underset{\nu \rightarrow 0}{=} \frac{\hbar c \pi}{2 a^{2}}\left[\sum_{n=1}^{\infty} e^{-\nu n} n\left(\sqrt{1+\left(\beta^{*} n\right)^{2}}+\beta^{*} n\right)\right. \\
\left.-\int_{0}^{\infty} e^{-\nu n} n\left(\sqrt{1+\left(\beta^{*} n\right)^{2}}+\beta^{*} n\right) d n\right]
\end{aligned}
$$

where the dimensionless deformation parameter is introduced

$$
\beta^{*}=\beta \frac{\hbar c \pi}{2 a} .
$$

Let us now apply the Abel-Plana formula [9] to calculate the sum over $n$ in Eq. (9):

$$
\sum_{n=1}^{\infty} f(n)=\int_{0}^{\infty} f(n) d n-\frac{f(0)}{2}+i \int_{0}^{\infty} \frac{f(i t)-f(-i t)}{e^{2 \pi t}-1} d t .
$$

We have

$$
f(n)=e^{-\nu n} n\left(\sqrt{1+\left(\beta^{*} n\right)^{2}}+\beta^{*} n\right) .
$$

Now, taking into account (10), the integrals over $n$ in Eq. (9) cancel out, $f(0)=0$, and the integration over $t$ (with the branching of the integrand at $t=1 / \beta^{*}$ taken into consideration) gives:

$$
\varepsilon=-\frac{\hbar c \pi}{a^{2}} \int_{0}^{1 / \beta^{*}} \frac{t \sqrt{1-\left(\beta^{*} t\right)^{2}}}{e^{2 \pi t}-1} d t .
$$

The value $\beta^{*}=0$ leads to the known result $[8,10]$ :

$$
\varepsilon=-\frac{\hbar c \pi}{a^{2}} \int_{0}^{\infty} \frac{t}{e^{2 \pi t}-1} d t=-\frac{\hbar c \pi}{24 a^{2}} .
$$


Let us now find the expansion of $\varepsilon$ at $\beta^{*}>1$. For this purpose one can write expression (12) as follows:

$$
\varepsilon=-\frac{\hbar c}{2 \beta^{*} a^{2}} \int_{0}^{1} \sqrt{1-x^{2}} \frac{2 \pi x / \beta^{*}}{e^{2 \pi x / \beta^{*}}-1} d x .
$$

Let us expand the second factor under the integral over the powers of $2 \pi x / \beta^{*}$ using the definition of the Bernoulli numbers $B_{n}$ [11, 12]:

$$
\varepsilon=-\frac{\hbar c}{2 \beta^{*} a^{2}} \sum_{n=0}^{\infty} \frac{B_{n}}{n !}\left(\frac{2 \pi}{\beta^{*}}\right)^{n} \int_{0}^{1} x^{n} \sqrt{1-x^{2}} d x .
$$

This integral is Euler's B-function, and the Bernoulli numbers are expressed via Riemann's $\zeta$-function [11, 12]. As a result we obtain:

$$
\begin{aligned}
\varepsilon & =\frac{\hbar c \pi}{2 a^{2}}\left(\sum_{n=1}^{\infty}(-)^{n} \frac{\Gamma(n+1 / 2) \zeta(2 n)}{(n+1) ! 2 \sqrt{\pi} \beta^{* 2 n+1}}-\frac{1}{4 \beta^{*}}+\frac{1}{3 \beta^{* 2}}\right) \\
& =-\frac{\hbar c \pi}{24 a^{2}}\left(\frac{3}{\beta^{*}}-\frac{4}{\beta^{* 2}}+\frac{\pi^{2}}{4 \beta^{* 3}}-\frac{\pi^{4}}{120 \beta^{* 5}}+\frac{\pi^{6}}{2016 \beta^{* 7}}+\ldots\right) .
\end{aligned}
$$

It is interesting to find this result directly from Eq. (9). For this purpose, let us single out the asymptotics $2 \beta^{*} n^{2}+1 / 2 \beta^{*}, n \rightarrow \infty$, of the expression in parentheses in formula (9) and write it as follows:

$$
\begin{aligned}
\varepsilon \underset{\nu \rightarrow 0}{=} \frac{\hbar c \pi}{2 a^{2}}\left\{\sum_{n=1}^{\infty} e^{-\nu n}\left[\beta^{*} n^{2}\left(\sqrt{1+\frac{1}{\left(\beta^{*} n\right)^{2}}}-1\right)-\frac{1}{2 \beta^{*}}\right]\right. \\
-\int_{0}^{\infty} e^{-\nu n}\left[\beta^{*} n^{2}\left(\sqrt{1+\frac{1}{\left(\beta^{*} n\right)^{2}}}-1\right)-\frac{1}{2 \beta^{*}}\right] d n \\
\left.+\sum_{n=1}^{\infty} e^{-\nu n}\left(2 \beta^{*} n^{2}+\frac{1}{2 \beta^{*}}\right)-\int_{0}^{\infty} e^{-\nu n}\left(2 \beta^{*} n^{2}+\frac{1}{2 \beta^{*}}\right) d n\right\} .
\end{aligned}
$$

At $\nu \rightarrow 0$ the first integral in this formula equals $\left(-1 / 3 \beta^{* 2}\right)$, and the difference of two last expressions equals $\left(-1 / 4 \beta^{*}\right)$. Finally we get

$$
\varepsilon=\frac{\hbar c \pi}{2 a^{2}}\left\{\sum_{n=1}^{\infty}\left[\beta^{*} n^{2}\left(\sqrt{1+\frac{1}{\left(\beta^{*} n\right)^{2}}}-1\right)-\frac{1}{2 \beta^{*}}\right]-\frac{1}{4 \beta^{*}}+\frac{1}{3 \beta^{* 2}}\right\} .
$$

As the sum over $n$ converges, the cut-off parameter $\nu$ can be put zero. Proceeding further with a formal expansion of the square root in the series of $1 / \beta^{* 2}$ and using the definition of Riemann's $\zeta$-function, we come to (16).

To make the computer calculation more convenient, formula (18) can be transformed to the following form: 


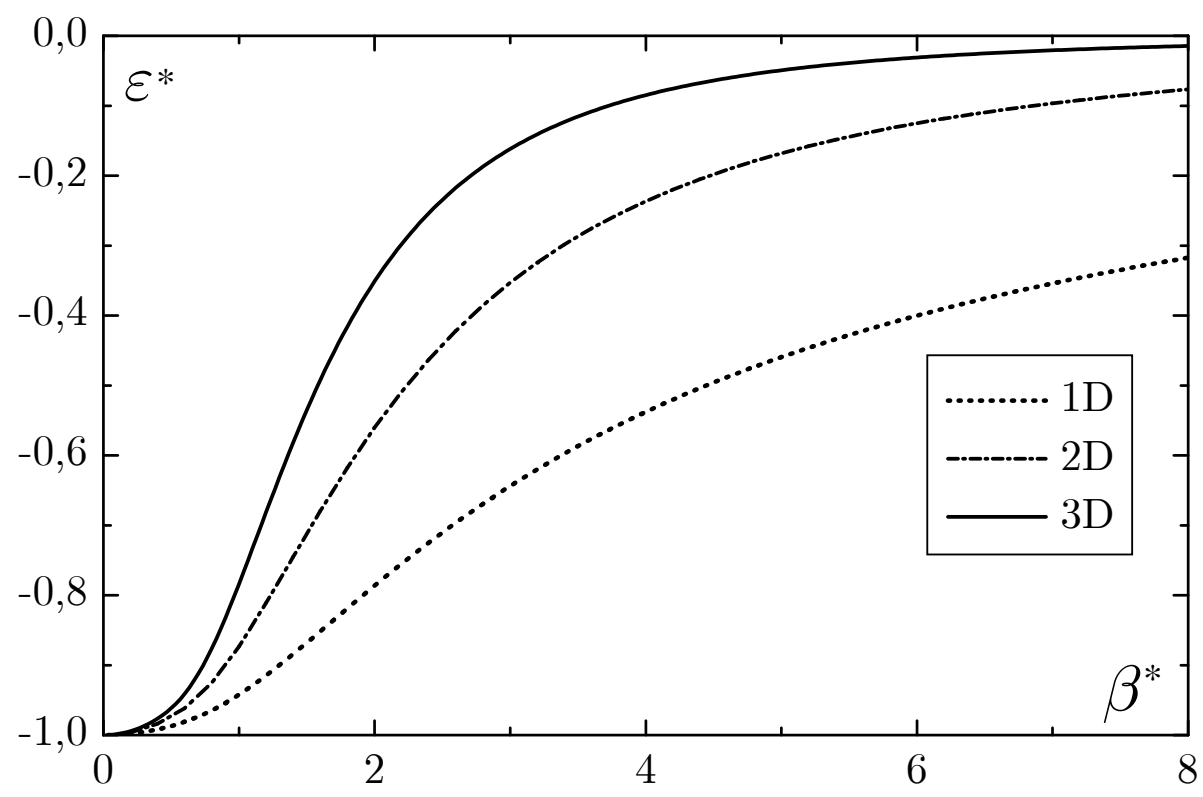

Figure 1: Casimir energy as a function of the deformation parameter.

$$
\varepsilon=-\frac{\hbar c \pi}{2 \beta^{*} a^{2}}\left[\frac{1}{2} \sum_{n=1}^{\infty} \frac{1}{\left(\sqrt{1+\left(\beta^{*} n\right)^{2}}+\beta^{*} n\right)^{2}}+\frac{1}{4}-\frac{1}{3 \beta^{*}}\right] .
$$

It should be noted, however, that the calculation of $\varepsilon$ using (12) is more effective in comparison with the summation in (19). This is the consequence of a fast convergence of the integral in (12).

Let us now make the expansion of the Casimir energy at small values of the deformation parameter. One can apply the Euler-Maclaurin formula directly to (9), as Casimir did in [1]. However, the result can be obtained in a shorter way if expression (9) is written as follows:

$$
\begin{gathered}
\varepsilon \underset{\nu \rightarrow 0}{=}-\frac{\hbar c \pi}{2 a^{2}} \frac{d}{d \nu} \sqrt{1+\left(\beta^{*} \frac{d}{d \nu}\right)^{2}}\left(\sum_{n=1}^{\infty} e^{-\nu n}-\int_{0}^{\infty} e^{-\nu n} d n\right) \\
\underset{\nu \rightarrow 0}{=}-\frac{\hbar c \pi}{2 a^{2}} \frac{d}{d \nu} \sqrt{1+\left(\beta^{*} \frac{d}{d \nu}\right)^{2}}\left(\frac{1}{e^{\nu}-1}-\frac{1}{\nu}\right) \\
\underset{\nu \rightarrow 0}{=}-\frac{\hbar c \pi}{2 a^{2}} \frac{d}{d \nu} \sqrt{1+\left(\beta^{*} \frac{d}{d \nu}\right)^{2}} \sum_{n=1}^{\infty} \frac{B_{n}}{n !} \nu^{n-1} .
\end{gathered}
$$

Here we again used the definition of the Bernoulli numbers $B_{n}$ 11, 12. Let us now make a formal expansion of the square root over the powers of $\beta^{* 2}$, take the derivatives over $\nu$ at $\nu \rightarrow 0$ and after simple transformations find the 
following asymptotic series:

$$
\begin{aligned}
\varepsilon & =-\frac{\hbar c \pi}{2 a^{2}}\left(\frac{1}{12}+\sum_{n=0}^{\infty}(-)^{n} \frac{\Gamma(n-1 / 2)}{(n+1) ! 4 \sqrt{\pi}} B_{2 n+2} \beta^{* 2 n}\right) \\
& =-\frac{\hbar c \pi}{24 a^{2}}\left(1-\frac{\beta^{* 2}}{20}-\frac{\beta^{* 4}}{168}-\frac{\beta^{* 6}}{320}-\frac{5}{1408} \beta^{* 8}-\ldots\right) .
\end{aligned}
$$

The results of the calculation of the Casimir energy (12), (19)

$$
\varepsilon_{1 \mathrm{D}}^{*}=\varepsilon / \frac{\hbar c \pi}{24 a^{2}}
$$

are presented in Fig. 1 (curve 1D). As one can see, the Casimir energy for all the values of the parameter $\beta^{*}$ is larger than that of the undeformed field and tends to zero at $\beta^{*} \rightarrow \infty$. Therefore, the deformation leads to the decrease of the attraction of the domain boundaries localizing the field caused by the polarization of a vacuum.

\section{A three-dimensional case}

Let us now calculate the Casimir energy for the field concentrated is a three-dimensional (3D) space between two infinite parallel plates separated by the distance $a$. The field equals zero on the plates. The wave vec-

tor $k=\sqrt{k_{x}^{2}+k_{y}^{2}+k_{z}^{2}},-\infty<k_{x}<\infty,-\infty<k_{y}<\infty, k_{z}=\pi n / a$, $n=0,1,2, \ldots$, and the sum

$$
\sum_{\mathbf{k}} \ldots \rightarrow \frac{1}{(2 \pi)^{2}} \int_{-\infty}^{\infty} d k_{x} \int_{-\infty}^{\infty} d k_{y} \sum_{n=0}^{\infty} \ldots
$$

Unlike the $1 \mathrm{D}$ case, in three dimensions the term with $n=0$ must be taken into account in the expression for the ground state energy (7). However, the polarization index $\alpha$ in this case has only one value which is a consequence of the field transversality. Therefore, by definition the Casimir energy equals

$$
\begin{aligned}
\varepsilon & =\frac{1}{a} \frac{1}{(2 \pi)^{2}} \int_{-\infty}^{\infty} d k_{x} \int_{-\infty}^{\infty} d k_{y} \frac{\hbar c}{2} \sqrt{k_{x}^{2}+k_{y}^{2}} \\
& \times\left[\sqrt{1+\left(\frac{\beta \hbar c}{2}\right)^{2}\left(k_{x}^{2}+k_{y}^{2}\right)}+\frac{\beta \hbar c}{2} \sqrt{k_{x}^{2}+k_{y}^{2}}\right] \\
& +\frac{1}{a} \sum_{\alpha} \frac{1}{(2 \pi)^{2}} \int_{-\infty}^{\infty} d k_{x} \int_{-\infty}^{\infty} d k_{y} \sum_{n=1}^{\infty} \frac{\hbar c}{2} \sqrt{k_{x}^{2}+k_{y}^{2}+\left(\frac{\pi}{a} n\right)^{2}} \\
& \times\left[\sqrt{1+\left(\frac{\beta \hbar c}{2}\right)^{2}\left(k_{x}^{2}+k_{y}^{2}+\left(\frac{\pi}{a} n\right)^{2}\right)}+\frac{\beta \hbar c}{2} \sqrt{k_{x}^{2}+k_{y}^{2}+\left(\frac{\pi}{a} n\right)^{2}}\right] 22
\end{aligned}
$$




$$
\begin{aligned}
& -\sum_{\alpha} \frac{1}{(2 \pi)^{3}} \int_{-\infty}^{\infty} d k_{x} \int_{-\infty}^{\infty} d k_{y} \int_{-\infty}^{\infty} d k_{z} \frac{\hbar c}{2} \sqrt{k_{x}^{2}+k_{y}^{2}+k_{z}^{2}} \\
& \times\left[\sqrt{1+\left(\frac{\beta \hbar c}{2}\right)^{2}\left(k_{x}^{2}+k_{y}^{2}+k_{z}^{2}\right)}+\frac{\beta \hbar c}{2} \sqrt{k_{x}^{2}+k_{y}^{2}+k_{z}^{2}}\right]
\end{aligned}
$$

The integration is made in polar coordinates, $q=\sqrt{k_{x}^{2}+k_{y}^{2}}$, with a further change of variables $k=\sqrt{q^{2}+(\pi n / a)^{2}}$, replacing $k$ with $q$, and introducing the cut-off function $e^{-\nu q}$. The result of these transformations is as follows:

$$
\begin{aligned}
\varepsilon \underset{\nu \rightarrow 0}{=} \frac{\hbar c}{2 \pi a}\left\{\frac{1}{2} \int_{0}^{\infty} d q q^{2} e^{-\nu q}\left[\sqrt{1+\left(\frac{\beta \hbar c q}{2}\right)^{2}}+\frac{\beta \hbar c q}{2}\right]\right. \\
\left.+\sum_{n=1}^{\infty} \int_{\pi n / 2}^{\infty} d q q^{2} e^{-\nu q}\left[\sqrt{1+\left(\frac{\beta \hbar c q}{2}\right)^{2}}+\frac{\beta \hbar c q}{2}\right]\right\} \\
-\frac{\hbar c}{2 \pi^{2}} \int_{0}^{\infty} d k_{z} \int_{k_{z}}^{\infty} d q q^{2} e^{-\nu q}\left[\sqrt{1+\left(\frac{\beta \hbar c q}{2}\right)^{2}}+\frac{\beta \hbar c q}{2}\right] .
\end{aligned}
$$

The Abel-Plana formula (10) with the function

$$
f(n)=\int_{\pi n / 2}^{\infty} q^{2} e^{-\nu q}\left[\sqrt{1+\left(\frac{\beta \hbar c q}{2}\right)^{2}}+\frac{\beta \hbar c q}{2}\right] d q .
$$

is applied to the second term in braces. It is now seen that the term with $f(0)$ from Eq. (10) cancels the first term in (23), and the last term in (23) cancels the first integral from the Abel-Plana formula (10). After a simple transformation we obtain:

$$
\varepsilon=-\frac{\hbar c \pi^{2}}{2 a^{4}} \int_{0}^{1 / \beta^{*}} d t \frac{1}{e^{2 \pi t}-1} \int_{-t}^{t} x^{2} \sqrt{1-\left(\beta^{*} x\right)^{2}} d x .
$$

Taking the integral over $x$ we finally find:

$$
\varepsilon=-\frac{\hbar c \pi^{2}}{8 \beta^{* 2} a^{4}} \int_{0}^{1 / \beta^{*}} \frac{\left(2 \beta^{* 2} t^{3}-t\right) \sqrt{1-\left(\beta^{*} t\right)^{2}}+\arcsin \left(\beta^{*} t\right) / \beta^{*}}{e^{2 \pi t}-1} d t .
$$

At $\beta^{*} \rightarrow 0$ the numerator of the integrand in (26) tends to $8 \beta^{* 2} t^{3} / 3$ and

$$
\varepsilon=-\frac{\hbar c \pi^{2}}{3 a^{4}} \int_{0}^{\infty} \frac{t^{3}}{e^{2 \pi t}-1} d t=-\frac{\hbar c \pi^{2}}{720 a^{4}} .
$$

This is Casimir's result [1]. 
Let us now find the expansion of $\varepsilon$ over the powers of $1 / \beta^{*}$ at $\beta^{*} \neq 0$. Expression (26) after the change of variable $x=\beta^{*} t$ is written as follows:

$$
\varepsilon=-\frac{\hbar c \pi}{16 a^{4} \beta^{* 3}} \int_{0}^{1}\left[\left(2 x^{2}-1\right) \sqrt{1-x^{2}}+\frac{\arcsin x}{x}\right] \frac{2 \pi x / \beta^{*}}{e^{2 \pi x / \beta^{*}}-1} d x .
$$

Using the expansion of the second factor under the integral over the powers of $2 \pi x / \beta^{*}[11$, 12] we find:

$$
\varepsilon=-\frac{\hbar c \pi}{16 a^{4} \beta^{* 3}} \sum_{n=0}^{\infty} \frac{B_{n}}{n !}\left(\frac{2 \pi}{\beta^{*}}\right)^{n} I(n)
$$

where

$$
I(n)=\int_{0}^{1} x^{n}\left[\left(2 x^{2}-1\right) \sqrt{1-x^{2}}+\frac{\arcsin x}{x}\right] d x .
$$

The integration in (30) gives:

$$
\begin{aligned}
& I(0)=\frac{\pi}{2}\left(\ln 2-\frac{1}{4}\right) \\
& I(1)=\frac{\pi}{2}-\frac{16}{15} \\
& I(2 n)=\frac{\pi}{4 n}-\frac{2 \Gamma(3 / 2) \Gamma(n+3 / 2)}{n \Gamma(n+3)} .
\end{aligned}
$$

We need the values of $I(n)$ for $n>1$ only for even $n$ 's as starting from $B_{3}$ all the odd Bernoulli numbers equal zero. Using the connection of the Bernoulli numbers with Riemann's $\zeta$-function, after some transformation we find for $\beta^{*} \neq 0$ :

$$
\begin{aligned}
\varepsilon & =-\frac{\hbar c \pi^{2}}{16 a^{4} \beta^{* 3}}\left\{\frac{1}{2}\left(\ln 2-\frac{1}{4}\right)-\frac{1}{\beta^{*}}\left(\frac{\pi}{2}-\frac{16}{15}\right)\right. \\
& \left.+\sum_{n=1}^{\infty}(-)^{n-1} \frac{\zeta(2 n)}{\beta^{* 2 n} 2 n}\left[1-\frac{(2 n+1) !}{2^{2 n-1}(n !)^{2}(n+1)(n+2)}\right]\right\} \\
& =-\frac{\hbar c \pi^{2}}{16 a^{4} \beta^{* 3}}\left[\frac{1}{2}\left(\ln 2-\frac{1}{4}\right)-\frac{1}{\beta^{*}}\left(\frac{\pi}{2}-\frac{16}{15}\right)+\frac{\pi^{2}}{24 \beta^{* 2}}+\ldots\right]
\end{aligned}
$$

Let us now find the asymptotic expansion of $\varepsilon$ for small values of the parameter $\beta$ proceeding from formula (23) written as follows:

$$
\begin{aligned}
\varepsilon \underset{\nu \rightarrow 0}{=} \frac{d^{2}}{d \nu^{2}}\left[\sqrt{1+\left(\frac{\beta \hbar c}{2} \frac{d}{d \nu}\right)^{2}}-\frac{\beta \hbar c}{2} \frac{d}{d \nu}\right] \\
\quad \times \frac{1}{\nu}\left(\frac{\hbar c}{4 \pi a}+\frac{\hbar c}{2 \pi a} \sum_{n=1}^{\infty} e^{-\nu \pi n / a}-\frac{\hbar c}{2 \pi^{2}} \int_{0}^{\infty} d k_{z} e^{-\nu k_{z}}\right) .
\end{aligned}
$$


Changing the cut-off parameter $\nu^{\prime}=\nu \pi / a$ after simple calculations reverting further back from $\nu^{\prime}$ to $\nu$ we find:

$$
\varepsilon \underset{\nu \rightarrow 0}{=} \frac{\hbar c \pi^{2}}{2 a^{4}} \frac{d^{2}}{d \nu^{2}}\left[\sqrt{1+\left(\beta^{*} \frac{d}{d \nu}\right)^{2}}-\beta^{*} \frac{d}{d \nu}\right] \frac{1}{\nu}\left(\frac{1}{2}+\frac{1}{e^{\nu}-1}-\frac{1}{\nu}\right) .
$$

Let us make the expansion over small values of the parameter $\beta^{*}$, applying the procedure from the previous section. We obtain:

$$
\varepsilon=\frac{\hbar c \pi^{2}}{2 a^{4}}\left(-\frac{1}{360}+\sum_{k=1} \frac{\Gamma(k-1 / 2)(-)^{k-1} B_{2 k+4}}{k ! \sqrt{\pi} 2(2 k+4)(2 k+3)} \beta^{* 2 k}\right)
$$

or, writing explicitly,

$$
\varepsilon=-\frac{\hbar c \pi^{2}}{720 a^{4}}\left(1-\frac{1}{7} \beta^{* 2}-\frac{3}{112} \beta^{* 4}-\frac{5}{264} \beta^{* 6}+\ldots\right) .
$$

The first term gives Casimir's result [1] the remaining terms take into account the field deformation. As in a one-dimensional space, the deformation leads to the repulsion and thus diminishes the attraction between the plates.

The numerical calculation of the Casimir energy

$$
\varepsilon_{3 \mathrm{D}}^{*}=\varepsilon / \frac{\hbar c \pi^{2}}{720 a^{4}}
$$

from formula (26) is presented in Fig. 1 (curve 3D).

\section{A two-dimensional case}

Let in the two-dimensional (2D) $x y$ plane the field be concentrated in an infinite stripe of width $a$ between the straight lines $y=0$ and $y=a$, at which it equals zero. From the field transversality only one polarization remains. It is perpendicular to the wave vector with the components $k_{x}, k_{y}:-\infty<k_{x}<\infty$, $k_{y}=\pi n / a, n=1,2,3, \ldots$. The value $n=0$ is not taken into account, as at $n=0$ the field equals zero owing to its transversality.

The Casimir energy equals:

$$
\begin{aligned}
\varepsilon & =\frac{1}{2 \pi a} \int_{-\infty}^{\infty} d k_{x} \sum_{n=1}^{\infty} \frac{\hbar c}{2} \sqrt{k_{x}^{2}+\left(\frac{\pi}{a} n\right)^{2}} \\
& \times\left[\sqrt{1+\left(\frac{\beta \hbar c}{2}\right)^{2}\left(k_{x}^{2}+\left(\frac{\pi}{a} n\right)^{2}\right)}+\frac{\beta \hbar c}{2} \sqrt{k_{x}^{2}+\left(\frac{\pi}{a} n\right)^{2}}\right] \\
& -\frac{1}{(2 \pi)^{2}} \int_{-\infty}^{\infty} d k_{x} \int_{-\infty}^{\infty} d k_{y} \frac{\hbar c}{2} \sqrt{k_{x}^{2}+k_{y}^{2}} \\
& \times\left[\sqrt{1+\left(\frac{\beta \hbar c}{2}\right)^{2}\left(k_{x}^{2}+k_{y}^{2}\right)}+\frac{\beta \hbar c}{2} \sqrt{k_{x}^{2}+k_{y}^{2}}\right] .
\end{aligned}
$$


As at $n=0$ the field is absent, the function $f(n)$ must be determined in such a way that $f(0)=0$. This determination can be made through the cut-off function $e^{-\nu / n}$. The same determination must be made also in the second term of Eq. (36). This corresponds to the field energy in a large volume tending to infinity.

Using the Abel-Plana formula after simple transformations similar to those made in the previous section we obtain from (36):

$$
\varepsilon=-\frac{\hbar c \pi}{a^{3}} \int_{0}^{1 / \beta^{*}} \frac{t^{2} d t}{e^{2 \pi t}-1} \int_{0}^{1} x^{2} \sqrt{\frac{1-\left(\beta^{*} t\right)^{2} x^{2}}{1-x^{2}}} d x
$$

At $\beta=0$ the integral over $x$ is easily calculated, it equals $\pi / 4$, and from (37) one obtains the known result [8, 10]:

$$
\varepsilon=-\frac{\hbar c}{16 \pi a^{3}} \zeta(3)
$$

At $\beta \neq 0$ the integral over $x$ in (37) cannot be expressed in elementary functions. It is reduced to complete elliptic integrals $E(k)$ and $K(k)$ [11, 12.

$$
\int_{0}^{1} x^{2} \sqrt{\frac{1-\left(\beta^{*} t\right)^{2} x^{2}}{1-x^{2}}} d x=\frac{1}{3}\left(2-\frac{1}{k^{2}}\right) E(k)+\frac{1}{3}\left(\frac{1}{k^{2}}-1\right) K(k),
$$

where the modulus $k=\beta^{*} t$.

Let us now find the expansion of $\varepsilon$ at small $\beta$. For this purpose, one can use the known expansions of elliptic integrals at small values of the modulus $k$ or proceed directly from Eq. (37). Thus, let us expand in (37) the square root in the numerator under the integral over $x$ in the series over $\beta^{*}$ :

$$
\varepsilon=-\frac{\hbar c \pi}{a^{3}} \sum_{m=0}^{\infty} \frac{\Gamma(3 / 2)\left(-\beta^{* 2}\right)^{m}}{m ! \Gamma(3 / 2-m)} \int_{0}^{1} d x \frac{x^{2 m+2}}{\sqrt{1-x^{2}}} \int_{0}^{1 / \beta^{*}} \frac{t^{2 m+2}}{e^{2 \pi t}-1} d t .
$$

The first integral over $x$ is equal to $\Gamma(m+3 / 2) \Gamma(1 / 2) / 2 \Gamma(m+2)$. The second integral over $t$ can be split into two parts: from 0 to $\infty$ and from $\infty$ to $1 / \beta^{*}$, the first one equaling $\Gamma(2 m+3) \zeta(2 m+3) / 2 \pi^{2 m+3}$ and the second one gives the terms proportional to $e^{-1 / \beta^{*}}$, thus we neglect it at $\beta^{*} \rightarrow 0$. Consequently, after simple transformations we obtain:

$$
\begin{aligned}
\varepsilon & =-\frac{\hbar c \pi}{a^{3}}\left[\frac{\zeta(3)}{16 \pi^{2}}-\sum_{m=1} \frac{\Gamma(3 / 2) \Gamma(m-1 / 2) \Gamma(1 / 2) \Gamma(m+3 / 2)}{2 \pi \Gamma(m+2) m !}\right. \\
& \left.\times \frac{\Gamma(2 m+3) \zeta(2 m+3)}{(2 \pi)^{2 m+3}} \beta^{* 2 m}+O\left(e^{-1 / \beta^{*}}\right)\right] \\
& =-\frac{\hbar c \pi}{a^{3}}\left[\frac{\zeta(3)}{16 \pi^{2}}-\frac{9 \zeta(5)}{128 \pi^{4}} \beta^{* 2}-\frac{225 \zeta(7)}{2048 \pi^{6}} \beta^{* 4}+\ldots\right]
\end{aligned}
$$


Let us proceed to the expansion of the Casimir energy over the inverse powers of the deformation parameter. It is simpler to do this directly from formula (37) changing the variable of integration $y=\beta^{*} t$ and expanding the integrand over the powers of $1 / \beta^{*}$ as it was done in the previous cases:

$$
\varepsilon=-\frac{\hbar c}{2 a^{3} \beta^{* 2}} \sum_{n=0}^{\infty} \frac{B_{n}}{n !}\left(\frac{2 \pi}{\beta^{*}}\right)^{n} J(n),
$$

where

$$
J(n)=\int_{0}^{1} d x \frac{x^{2}}{\sqrt{1-x^{2}}} \int_{0}^{1} d y y^{n+1} \sqrt{1-x^{2} y^{2}} .
$$

The quantities $J(n)$ for specific values of $n=0,1,2, \ldots$ can be easily calculated:

$$
\begin{aligned}
& J(0)=\frac{1}{3}\left(\frac{\pi}{2}-\frac{2}{3}\right), \\
& J(1)=\frac{G}{4}-\frac{1}{24}, \\
& J(2)=\frac{2}{15},
\end{aligned}
$$

here $G=0.915965594 \ldots$ is Catalan's constant. Finally at $\beta^{*} \neq 0$ we obtain:

$$
\varepsilon=-\frac{\hbar c}{2 a^{3} \beta^{* 2}}\left[\frac{1}{3}\left(\frac{\pi}{2}-\frac{2}{3}\right)-\left(\frac{G}{4}-\frac{1}{24}\right) \frac{\pi}{\beta^{*}}+\frac{2 \pi^{2}}{45 \beta^{* 2}}+\ldots\right] .
$$

The results of numerical calculations using formula (37) for the dimensionless Casimir energy

$$
\varepsilon_{2 \mathrm{D}}^{*}=\varepsilon / \frac{\hbar c}{16 \pi a^{3}} \zeta(3)
$$

are presented in Fig. 1 (curve 2D). As for the dimensions $D=1, D=3$, the Casimir energy $\varepsilon$ tends to zero from below at $\beta \rightarrow \infty$.

\section{Conclusions}

We have shown that the deformation of the field given by Eq. (2) leads to the suppression of the Casimir effect for the considered simple-topology surfaces localizing the field. The mechanism of this suppression is clear from the fact that minimal mean-square fluctuations of field strengths equal zero for the undeformed field and are greater than $\hbar^{2} \beta$ for every vibration mode in the case of the deformed field. This very fact leads to additional repulsion of the domain boundaries confining the filed.

The author appreciates interesting discussion with V. Tkachuk, Yu. Krynytskyi, T. Fityo, and A. Rovenchak. 


\section{References}

[1] H. B. G. Casimir, Proc. K. Ned. Akad. Wet. B51, 793 (1948).

[2] O. Panella, Phys. Rev. D 76, 045012 (2007).

[3] U. Harbach and S. Hossenfelder, Phys. Lett B 632, 379 (2006).

[4] Kh. Nouicer, J. Phys. A 38, 10027 (2005).

[5] A. Kempf, G. Mangano, and R. B. Mann, Phys. Rev. D 52, 1108 (1995).

[6] A. Camacho, Int. J. Mod. Phys. D 12, 1687 (2003).

[7] V. I. Man'ko, G. Marmo, S. Solimeno, and F. Zaccaria, Phys. Lett. A 176, 173 (1993).

[8] I. O. Vakarchuk, Quantum mechanics, 3rd ed. (Lviv University Press, 2007) [in Ukrainian].

[9] M. A. Evgrafov, Analytic functions (Dover Publ., 1978).

[10] A. A. Grib, S. G. Mamaev, V. M. Mostepanenko, Vacuum quantum effects in the strong fields (Moscow, Energoatomizdat, 1988) [in Russian].

[11] E. Janke, F. Emde, Tables of Functions with Formulas and Curves, 4th ed. (Dover Publ., 1969).

[12] I. S. Gradshteyn, I. M. Ryzhik, Table of Integrals, Series, and Products, 5th ed. (Academic Press, 1994). 